Research article

urn:1sid:zoobank.org:pub:E42F1AE2-B8E6-43C8-896C-75A87814642A

\title{
Contribution to the Willowsia species having body scales of the long basal rib type: four new species and a redescription of W. qui (Collembola: Entomobryidae)
}

\author{
Zhixiang PAN ${ }^{1} \&$ Feng $\mathrm{ZHANG}^{2, *}$ \\ ${ }^{1}$ School of Life Sciences, Taizhou University, Jiaojiang, Taizhou 318000, Zhejiang Province, P. R. China. \\ ${ }^{2}$ Department of Entomology, College of Plant Protection, Nanjing Agricultural University, \\ 1 Weigang, Nanjing 210095, P. R. China. \\ ${ }^{1}$ Email: pzx1118@hotmail.com \\ *Corresponding author: xtmtd.zf@gmail.com \\ ${ }^{1}$ urn:Isid:zoobank.org:author:C66099CD-2681-453F-BCC3-3B627AB31DC5 \\ ${ }^{2}$ urn:1sid:zoobank.org:author:904C4445-AAEA-40BC-B418-502053EF39B8
}

\begin{abstract}
Four new species of the genus Willowsia Shoebotham, 1917 are described from China: W. fascia Zhang \& Pan sp. nov., W. pseudoplatani Zhang \& Pan sp. nov., W. pseudobuskii sp. nov., and W. similis sp. nov. Willowsia qui Zhang, Chen \& Deharveng, 2011 is re-described based on types and fresh material. All five species possess the pointed body scales with basal ribs longer than distal ones. These scales are absent on antennae, legs, ventral tube and furca. Colour pattern and dorsal chaetotaxy are the main diagnostic characters for these species. DNA barcodes of four species are also provided, with genetic distances compared. A key to the Willowsia species having scales of the long basal rib type is given.
\end{abstract}

Keywords. DNA barcodes, colour pattern, chaetotaxy, Entomobryinae, China.

Pan Z. \& Zhang F. 2016. Contribution to the Willowsia species having body scales of the long basal rib type: four new species and a redescription of W. qui (Collembola: Entomobryidae). European Journal of Taxonomy 245: 1-25. http://dx.doi.org/10.5852/ejt.2016.245

\section{Introduction}

Willowsia Shoebotham, 1917 is the largest scaled Entomobryinae genus, comprising 27 species worldwide (Bellinger et al. 1996-2016). Its members have body scales mostly pointed, with rough sculpture of very short spinules to long ribs (Zhang et al. 2011). Yoshii \& Suhardjono (1989) questioned the monophyly of Willowsia, as well as of Willowsiini Yoshii \& Suhardjono, 1989, whose polyphyly was recently demonstrated by molecular phylogeny (Zhang et al. 2014a, b, 2015). Zhang et al. (2011) divided the taxa of Willowsia into four species groups based on the scale sculpture: spinulate type, short rib type, long rib type, and uninterrupted ribbed type. Eleven species, including the generic type $W$. nigromaculata (Lubbock, 1873), possess scales of the long rib type. In this study, four new species of this group from China are described. Willowsia qui Zhang, Chen \& Deharveng, 2011 is re-described 
based on types and fresh material because of the incorrect designation of one paratype in the original description. A key to the species of Willowsia that have scales of the long basal rib type is given.

\section{Material and Methods}

Specimens were mounted in Hoyer's solution after clearing in Nesbitt's fluid, and were studied using a Nikon 80i phase contrast microscope. Photographs were taken with a Nikon DS-Fil camera mounted on Nikon SMZ1000 and 80i microscopes and were enhanced with Photoshop CS2 (Adobe Inc.). Dorsal cephalic chaetae are named after Jordana \& Baquero (2005), interocular chaetae after Mari-Mutt (1986), labial chaetae after Gisin (1967), and tergal S-chaetae after Zhang \& Deharveng (2015). Tergal chaetae are designated following Szeptycki (1979). The number of macrochaetae is given by half-tergite in the descriptions. Material is deposited in the collections of the Department of Entomology, College of Plant Protection, Nanjing Agricultural University (NJAU), Nanjing, China, the School of Life Sciences, Taizhou University (TZU), Zhejiang, China, and the Muséum national d'Histoire naturelle (MNHN), Paris, France.

To obtain DNA barcodes (mitochondrial COI fragment), DNA extraction, amplification, purification and sequencing followed Zhang et al. (2014c). Sequences were assembled in Sequencher 4.5 (Gene Codes Corporation, Ann Arbor, USA), and deposited in GenBank. A final 658 bp alignment was obtained. Genetic distances were calculated in MEGA 5.0 (Tamura et al. 2011), with the Kimura-2 parameter substitution model and pairwise deletion for gaps.

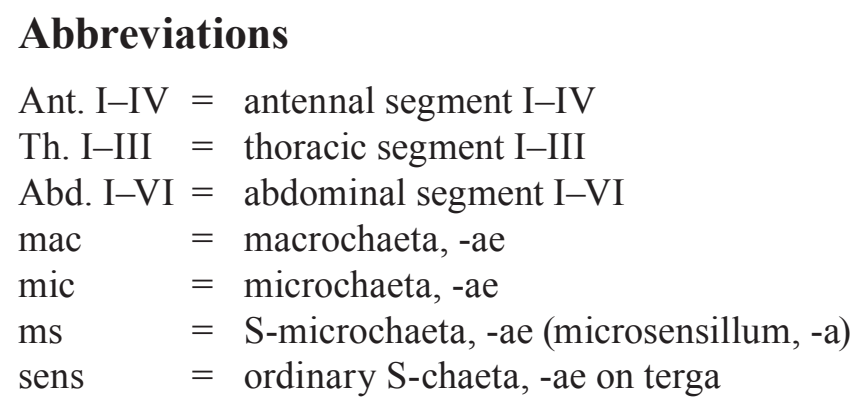

\section{Results}

The following characters are shared by the five species included in this paper and are not repeated in the descriptions: Ant. II distally with a small rod; Ant. III organ with 2 rods (Figs 2A, 8A, 11A, 14A); antennal apical bulb unilobed (Figs 7D, 10C); eyes 8+8, G and H smallest; labral margin with 4 conical papillae (Figs 2B, 5A); prelabral and labral chaetae 4/5, 5, 4, prelabral ones ciliate (Fig. 5A); mandibles with $4+5$ teeth; subapical chaeta of maxillary outer lobe larger than apical one, 3 hairs on sublobal plate (Figs 2E, 5C, 8D); interocular chaetae as pqrst (Fig. 2D); tip of lateral process of labial palp as thick as normal chaetae; labial chaetae $\mathrm{MREL}_{1} \mathrm{~L}_{2}$, all ciliate; chaetae posterior to labium ciliate; tibiotarsi distally with 10 chaetae in a whorl; unguis with 4 inner teeth; unguiculus lanceolate, with outer edge serrate; tenent hairs clavate; tenaculum with $4+4$ teeth and one large striate chaeta; mucro bidentate, its basal spine short, with tip reaching apex of subapical tooth (Figs 2L, 5J, 8O, 11I, 14J); accessory mic of bothriotrichal complexes on Abd. II-IV unmodified (Figs 1B, 7E, 7F, 13B); tergal ms as 1, 0|1, 0, 1, 0, 0,0 ; tergal sens as 2, 2|1, 2, 2, ?, 3, 0; ms on Abd. I anterior to sens; lateral sens as and ps on Abd. IV, obviously shorter than others. 


\author{
Class Collembola Lubbock, 1873 \\ Order Entomonryomorpha Börner, 1913 \\ Family Entomobryidae Tömösvary, 1882 \\ Genus Willowsia Shoebotham, 1917 \\ Willowsia fascia Zhang \& Pan sp. nov. \\ urn:Isid:zoobank.org:act:C5A9737F-4430-4D9A-89D5-54393C51A795
}

Figs 1-3; Table 1

\title{
Diagnosis
}

Transverse bands on Abd. II-III; cephalic mac $\mathrm{S}_{0}$ present; Th. II with $1(\mathrm{~m} 1)$ medio-median and $2(\mathrm{~m} 4$, m4i) medio-lateral, and 11 (p1-5, p1i, p2a, p2p, p2a, p2e, p3p) posterior mac; chaetae a2, a3, p5pi, p6pi on Th. III as mac; Abd. I with 3 (m2-4) mac; Abd. III with 3 (a2, a3, m3) central mac.

\section{Etymology}

Named after the dark bands on the abdomen.

\section{Material examined}

\section{Holotype}

CHINA: + on slide, Hubei Province, Yingshan County, Taohuachong Forest Park, $30.982^{\circ} \mathrm{N}, 116.020^{\circ} \mathrm{E}$, altitude 802 m, 24 Jun. 2014, F. Zhang and D.-Y. Yu leg. (slide \# 14HUB64_1, sample \# 14HUB242), deposited in NJAU.

\section{Paratypes}

CHINA: 3 $q+$ and $1 \delta^{\lambda}$ on slides and 2 in alcohol, same data as holotype. One paratype on slide in MNHN (slide \# 14HUB64_4) and others in NJAU (slides \# 14HUB64_1-3).
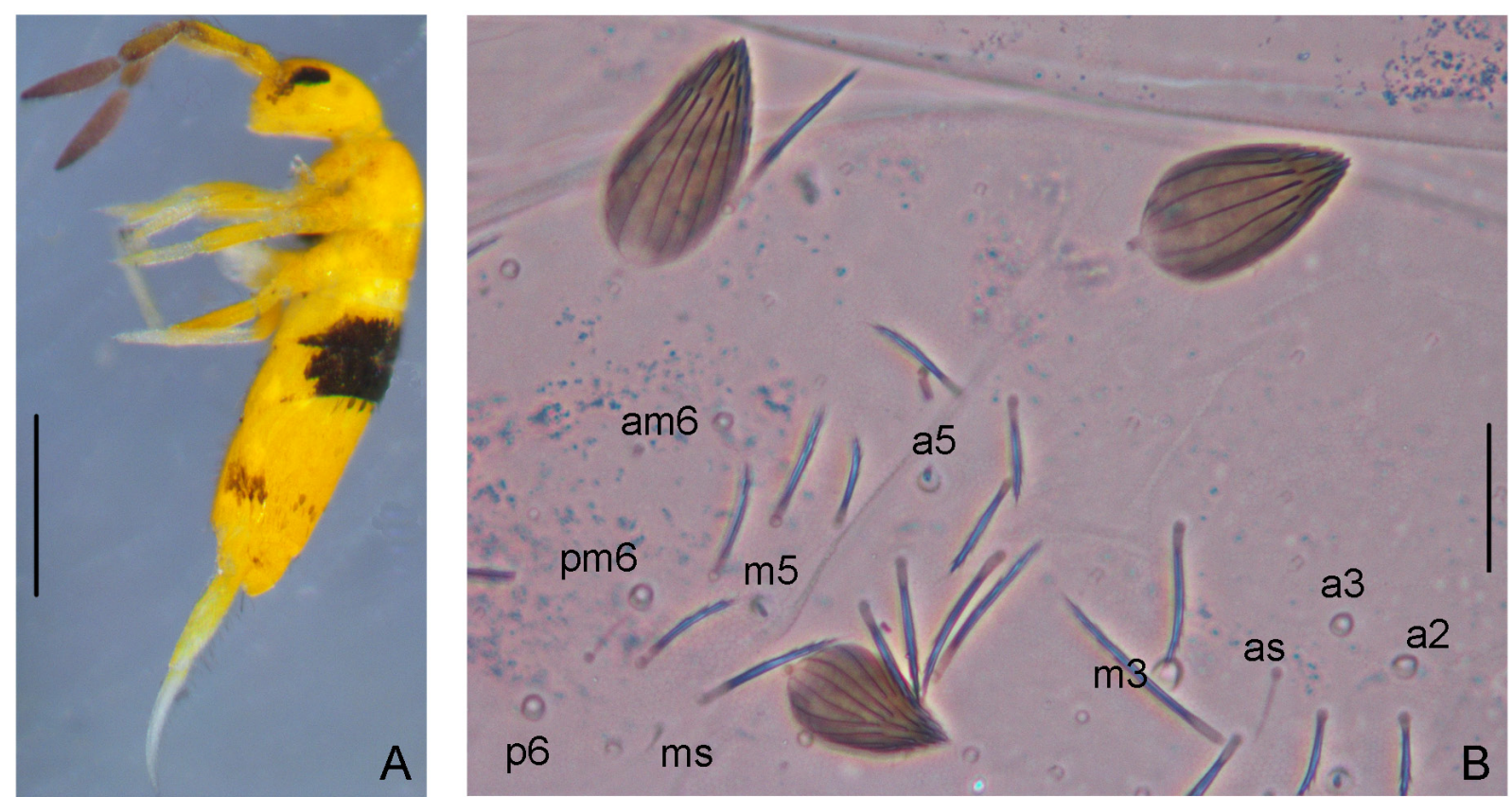

Fig. 1. Willowsia fascia Zhang \& Pan sp. nov. A. Habitus. B. Abd. III. Scale bars: A $=500 \mu \mathrm{m}$; B $=20 \mu \mathrm{m}$. 

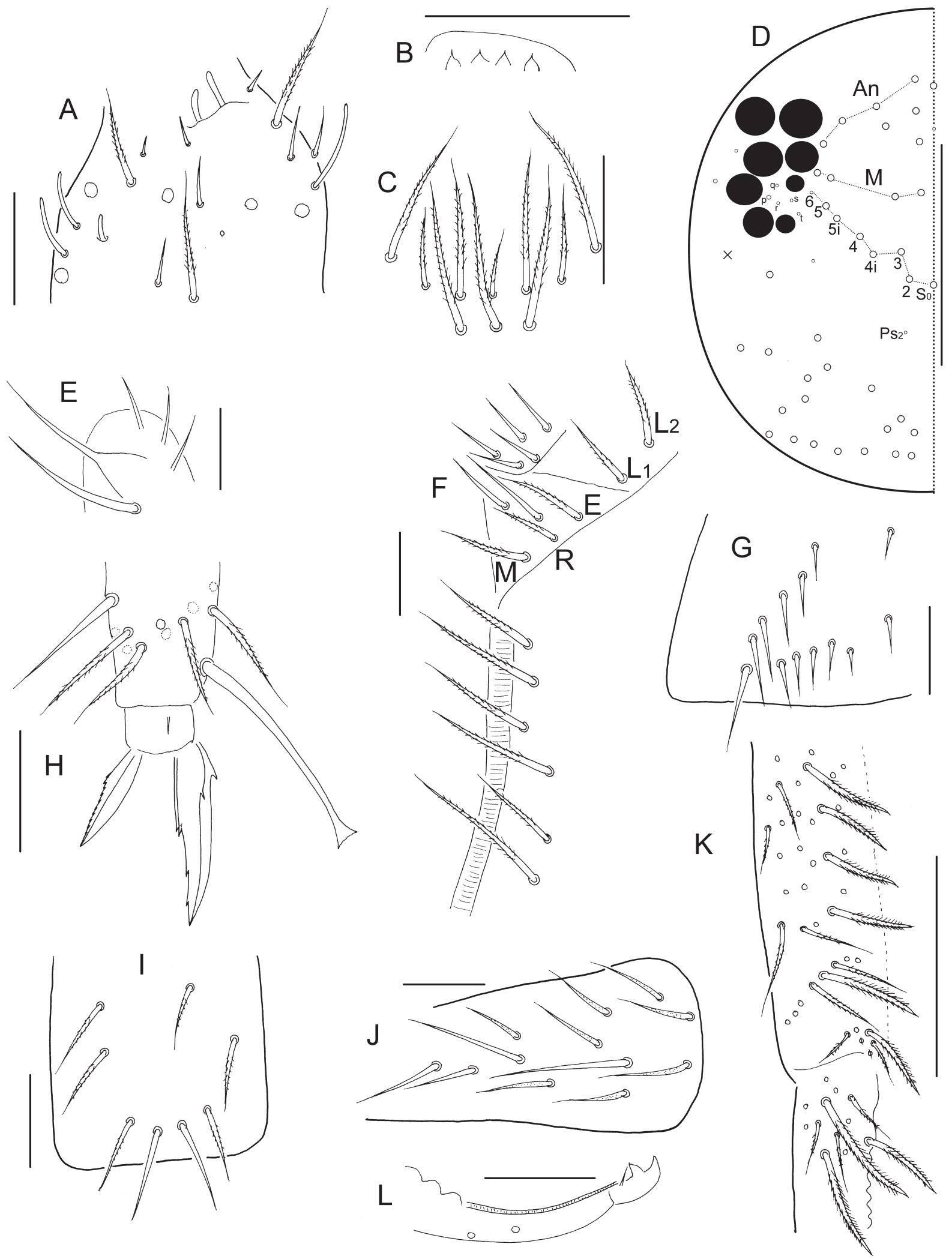

Fig. 2. Willowsia fascia Zhang \& Pan sp. nov. A. Ant. III organ. B. Labral papillae. C. Clypeal chaetae. D. Dorsal cephalic chaetotaxy. E. Maxillary outer lobe. F. Chaetae on ventral side of head. G. Trochanteral organ. H. Hind claw. I-J. Ventral tube. I. Posterior face. J. Lateral flap. K. Manubrium and base of dens, dorsal view. L. Mucro. Scale bars: A-C, E-J, L $=20 \mu \mathrm{m} ; \mathrm{D}, \mathrm{K}=100 \mu \mathrm{m}$. 

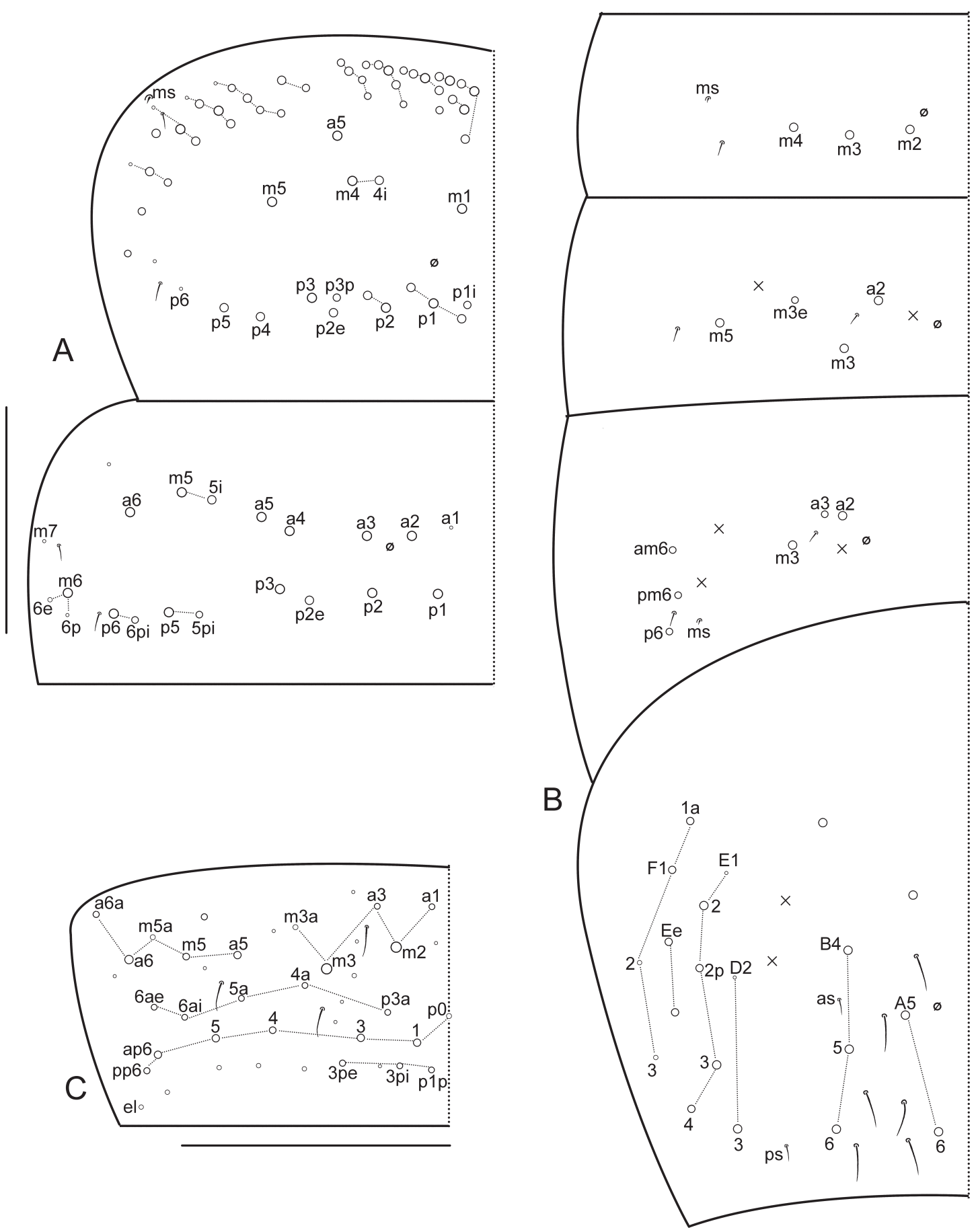

Fig. 3. Willowsia fascia Zhang \& Pan sp. nov. A. Thoracic chaetotaxy. B-C. Abdominal chaetotaxy. B. Abd. I-IV (long sens on Abd. IV incomplete). C. Abd. V. Scale bars: $100 \mu \mathrm{m}$. 
Table 1. Comparison between $W$. similis sp. nov. and W. fascia Zhang \& Pan sp. nov.

\begin{tabular}{lcc}
\hline Characters & W. fascia & W. similis \\
& Zhang \& Pan sp. nov. & sp. nov. \\
\hline Transverse band on metathorax & absent & present \\
$\mathrm{S}_{0}$ dorsally on head & present & absent \\
Chaeta p6 on Th. II & mic & mac \\
Mac a3, p5pi and p6pi on Th. III & present & absent \\
\hline
\end{tabular}

\section{Description}

Body length up to $1.70 \mathrm{~mm}$.

Ground colour yellow in alcohol. Ant. I-IV gradually darker. Transverse bands on Abd. II-III (Fig. 1A). Scales brown, pointed, heavily striate with basal ribs very long (Fig. 1B), and only present dorsally on head and terga. Partial chaetae of the row along the posterior margin of each tergum transformed into scales and larger than other scales.

Based on 6 measured specimens, antenna 2.43-2.53 times as long as cephalic diagonal. Antennal segment ratio as I: II: III: IV = 1: 1.87-2.06: 1.60-1.88: 2.67-2.81. Smooth spiny mic at base of antennae: 3 dorsal and 4 ventral on Ant. I; 1 internal, 1 external and 1 ventral on Ant. II.

Labral intrusion U-shaped. Clypeal chaetae 10 in number, all ciliate and 3 of them smaller (Fig. 2C). Dorsal cephalic chaetotaxy with 4 antennal (An), 4 median (M) and 7 sutural (S) mac; mac $\mathrm{S}_{1}$ absent; mac $S_{0}$ present; interocular chaeta $r$ absent on one side in one specimen. Tip of lateral process of labial palp just reaching or slightly beyond apex of labial papilla. Labial chaeta $\mathrm{R} / \mathrm{M}=0.55-0.70$. Cephalic groove with $6+6(5)$ ciliate chaetae (Fig. 2F).

Metatrochanteral organ with 8-13 smooth spine-like chaetae; 6-10 in L-shaped arms and 2-3 between arms (Fig. 2G). Ungual basal paired inner teeth with tip reaching $50 \%$ from base, median one at $74 \%$ and distal one at $87 \%$. Tenent hairs slightly longer than unguis in length (Fig. 2H). Abd. IV 2.54-3.95 times as long as Abd. III along dorsal midline. Ventral tube anteriorly not clearly seen; posteriorly with 6-8 chaetae, apical two smooth (Fig. 2I); each lateral flap with 8-12 chaetae and 3-4 of them weakly ciliate (Fig. 2J). Male genital plate papillate, not clearly seen. Manubrium and base of dens dorsally with 6 and 3 large differentiated chaetae, respectively. Manubrial plaque with 2 pseudopores and 4 ciliate chaetae (Fig. 2K). Distal smooth part of dens 1.62-2.60 times as long as mucro.

Th. II with $1(\mathrm{~m} 1)$ medio-medial and $2(\mathrm{~m} 4, \mathrm{~m} 4 \mathrm{i})$ medio-lateral, and 11 posterior mac; $\mathrm{m} 5$ as mac. Th. III with 16 mac; al as mic; p5pi and p6pi as mac (Fig. 3A). Abd. I with 3 (m2-4) mac. Abd. II with 3 (a2, m3, m3e) central and 1 (m5) lateral mac. Abd. III with 3 (a2, a3, m3) central and 3 (am6, pm6, p6) lateral mac. Abd. IV with 7 central and 9 lateral mac; F2 and F3 as mesochaetae (Fig. 3B).

\section{Ecology}

In decomposing leaves.

\section{DNA barcode}

KU833222. 


\section{Remarks}

Willowsia fascia sp. nov. is characterized by the two transverse bands on Abd. II-III. The new species is closest to $W$. similis sp. nov. in the dark Abd. II-III, scale sculpture, clypeal chaetae, labrum, labium, claw, mucro and abdominal chaetotaxy, and differs from the latter in the unpigmented metathorax, mac $S_{0}$ present dorsally on the head, and thoracic chaetotaxy (Table 1$)$. The great genetic distance $(0.200)$ of the mitochondrial COI also indicates two independent species.

Willowsia pseudoplatani Zhang \& Pan sp. nov. urn:Isid:zoobank.org:act:CFF949FE-57F5-40DC-8087-04920282F729

Figs 4-6

\section{Diagnosis}

Metathorax and Abd. II-IV dark blue; cephalic mac $\mathrm{S}_{0}$ and $\mathrm{S}_{1}$ absent; manubrial plaque with about 7 ciliate chaetae; Th. II with $1(\mathrm{~m} 4)$ medio-lateral and $6(\mathrm{p} 1-5, \mathrm{p} 2 \mathrm{e})$ posterior mac and without mediomedial mac; Th. III with mic a1 and a3; Abd. I with 3 (m2-4) mac; Abd. III with 2 (a2, m3) central mac.

\section{Etymology}

Named after the great similarities between the new species and W. platani (Nicolet, 1842).

\section{Material examined}

\section{Holotype}

CHINA: + on slide, Zhejiang Province, Taishun County, Wuyanling National Forest Park, Shuangkengkou, $27.617^{\circ} \mathrm{N}, 119.767^{\circ} \mathrm{E}$, altitude $600 \mathrm{~m}, 8$ Aug. 2010, JX Chen team leg. (slide \# C9279_1, sample \# C9279), deposited in NJAU.
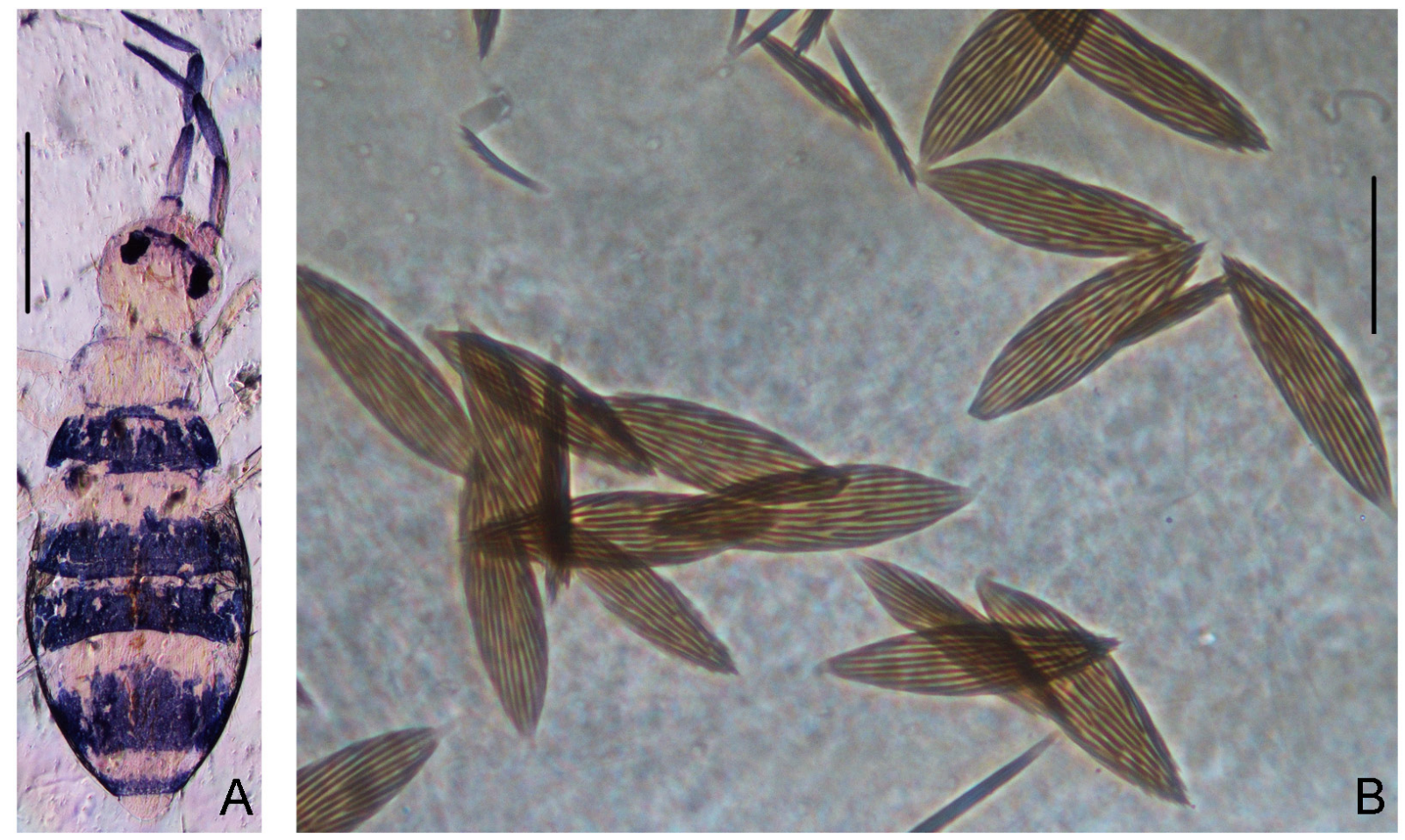

Fig. 4. Willowsia pseudoplatani Zhang \& Pan sp. nov. A. Colour pattern. B. Scales on Abd. II. Scale bars: $\mathrm{A}=500 \mu \mathrm{m} ; \mathrm{B}=20 \mu \mathrm{m}$. 

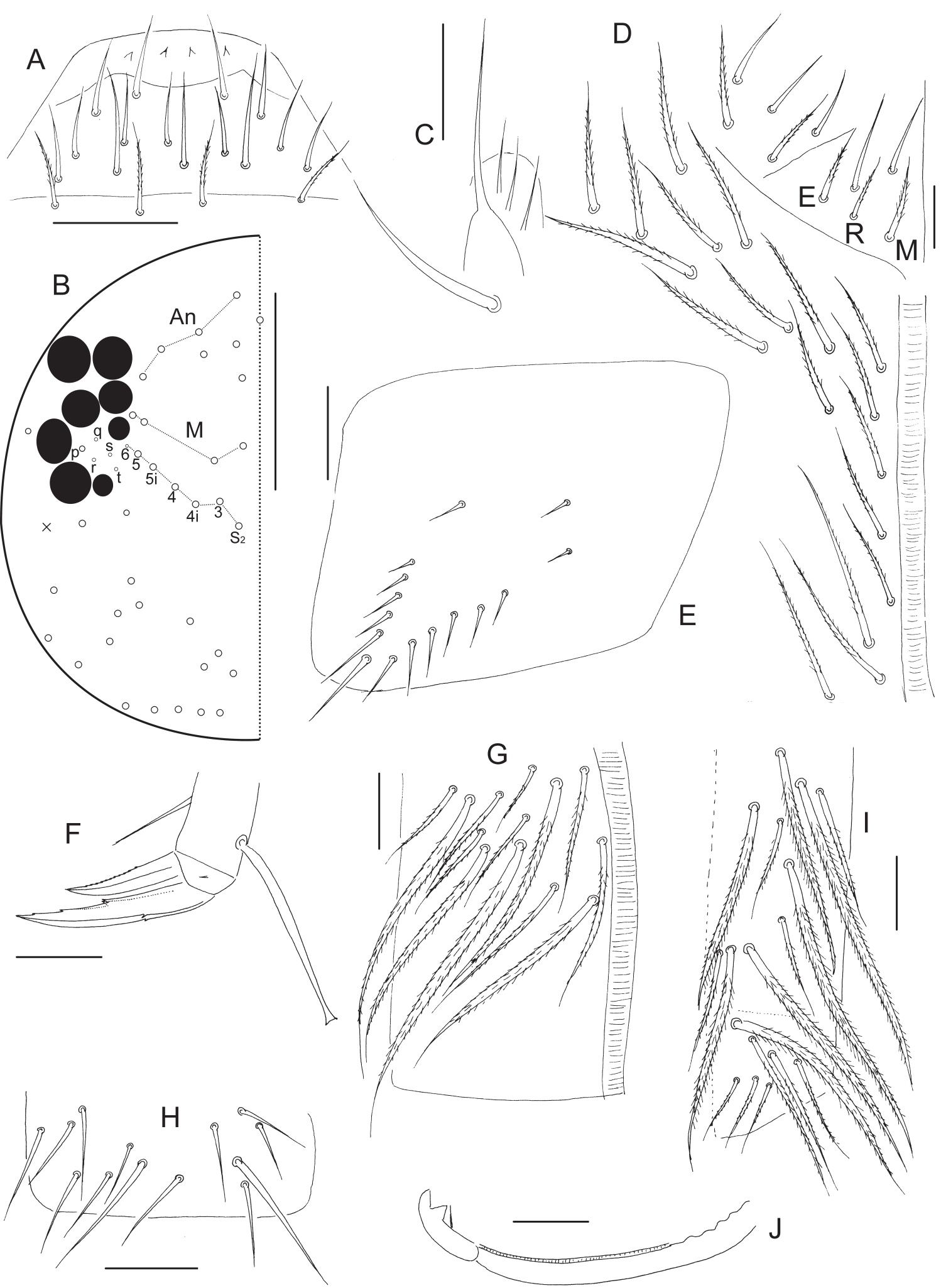

Fig. 5. Willowsia pseudoplatani Zhang \& Pan sp. nov. A. Labrum. B. Dorsal cephalic chaetotaxy. C. Maxillary outer lobe. D. Chaetae on ventral side of head. E. Trochanteral organ. F. Hind claw. G-H. Ventral tube. G. Anterior face. H. Lateral flap. I. Manubrium, dorsal view. J. Mucro. Scale bars: $\mathrm{A}, \mathrm{C}-\mathrm{J}=20 \mu \mathrm{m} ; \mathrm{B}=100 \mu \mathrm{m}$. 

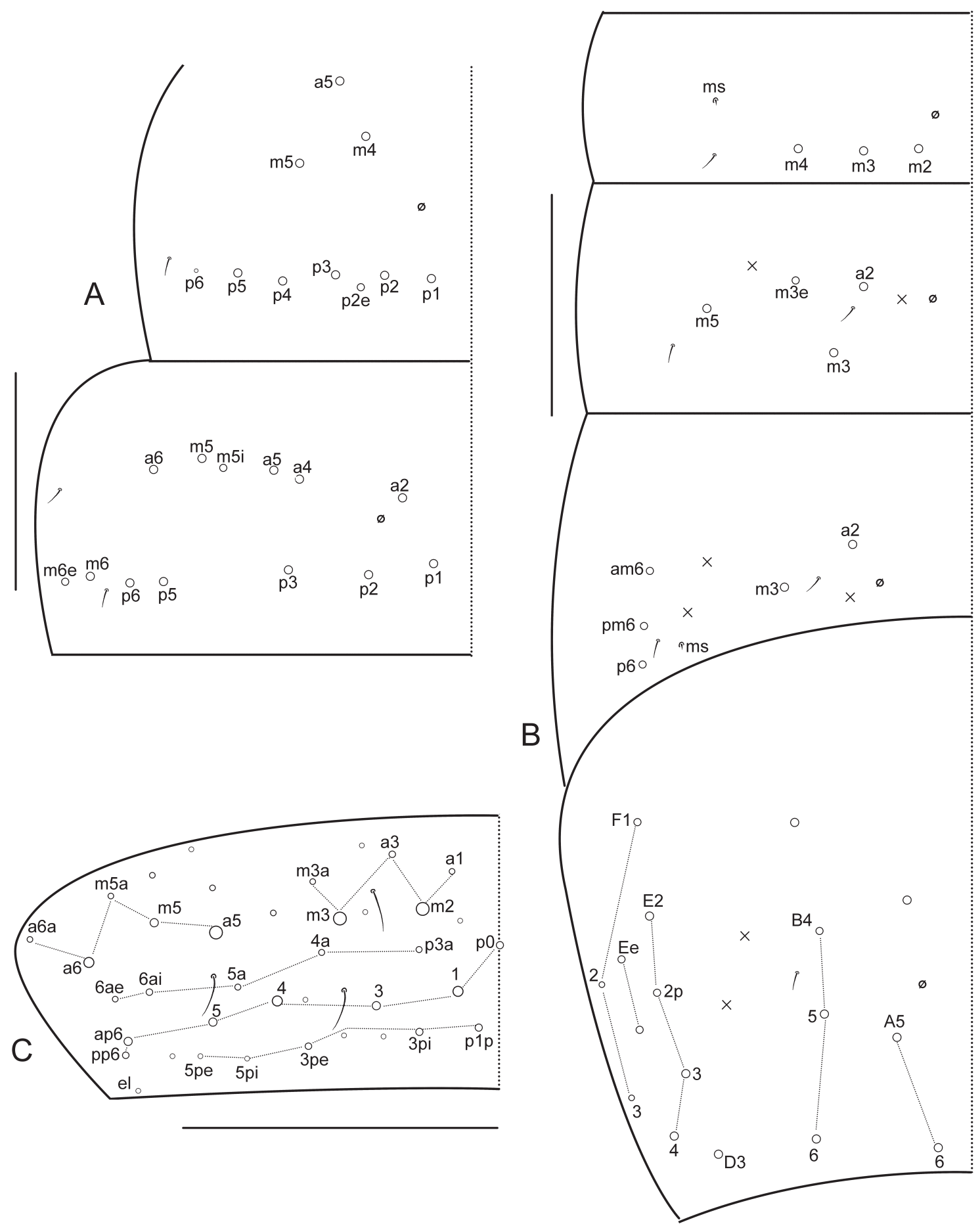

Fig. 6. Willowsia pseudoplatani Zhang \& Pan sp. nov. A. Thoracic chaetotaxy. B-C. Abdominal chaetotaxy. B. Abd. I-IV. C. Abd. V. Scale bars: $100 \mu \mathrm{m}$. 
Paratypes

CHINA: 2 ㅇ on slides, same data as holotype (slide \# C9279_2-3), deposited in NJAU.

\section{Description}

Body length up to $1.74 \mathrm{~mm}$.

Ground colour pale in alcohol. Ant. I-IV gradually darker. A transverse band between the eye patches. Dark blue stripe on lateral margin of mesothorax. Metathorax and Abd. II-IV dark blue. Abd. V posteriorly with a transverse band (Fig. 4A). Scales narrow, pointed, heavily striate with basal ribs longer than distal ones (Fig. 4B), and only present dorsally on head and terga.

Based on 3 measured specimens, antenna 2.14-2.30 times as long as cephalic diagonal. Antennal segment ratio as I: II: III: IV = 1: 2.17-2.40: 2.17-2.33: 3.00. Smooth spiny mic at base of antennae 3: dorsal and 3 ventral on Ant. I; 1 internal, 1 external and 1 ventral on Ant. II.

Labral intrusion slightly curved (Fig. 5A). Clypeal chaetae ciliate but their number not clearly seen. Dorsal cephalic chaetotaxy with 4 antennal (An), 4 median (M) and 6 sutural (S) mac; mac $\mathrm{S}_{0}$ and $\mathrm{S}_{1}$ absent (Fig. 5B). Tip of lateral process of labial palp not reaching apex of labial papilla. Labial chaeta $\mathrm{R} / \mathrm{M}=0.71-0.76$. Cephalic groove with 7-8 ciliate chaetae on each side (Fig. 5D).

Metatrochanteral organ with 11-16 smooth spine-like chaetae; 7-12 in L-shaped arms and 3-4 between arms (Fig. 5E). Ungual basal inner paired teeth with tip reaching $50 \%$ from base, median one at $75 \%$ and distal one at $86 \%$. Tenent hairs subequal to unguis in length (Fig. 5F). Abd. IV 3.04-3.95 times as long as Abd. III along dorsal midline. Ventral tube anteriorly with about 13 ciliate chaetae, 5 of them larger than others (Fig. 5G); posteriorly not clearly seen; each lateral flap with 10-13 chaetae, some of them weakly ciliate (Fig. 5H). Manubrium dorsally with $7+7$ large differentiated chaetae. Manubrial plaque with about 7 ciliate chaetae; pseudopores not clearly seen (Fig. 5I). Distal smooth part of dens 2.50-2.75 times as long as mucro.

Th. II with $1(\mathrm{~m} 4)$ medio-lateral and $6(\mathrm{p} 1-5, \mathrm{p} 2 \mathrm{e})$ posterior mac; medio-medial mac absent; $\mathrm{m} 5 \mathrm{as}$ mac. Th. III with 13 mac; a1 and a3 as mic (Fig. 6A). Abd. I with 3 (m2-4) mac. Abd. II with 3 (a2, m3, m3e) central and 1 (m5) lateral mac. Abd. III with 2 (a2, m3) central and 3 (am6, pm6, p6) lateral mac. Abd. IV with 7 central and about 8 lateral mac; F1 often as mic; F2 and F3 as mesochaetae (Fig. 6B).

\section{Ecology}

Found in litter, debris and rotten logs of evergreen broad-leaved forest.

\section{Remarks}

Willowsia pseudoplatani sp. nov. is characterized by the unique colour pattern and the thoracic chaetotaxy. The new species is closest to W. platani (Nicolet, 1842) in most features except several characters. W. pseudoplatani sp. nov. has pale Abd. V and longer smooth part of dens. In chaetotaxy, mac $\mathrm{m} 5$ on Th. II and mac a2, a5, m5i on Th. III are present in W. pseudoplatani sp. nov. but absent in W. platani; on Abd. III, a3 is mic in the new species and mac in W. platani. 


\section{Willowsia pseudobuskii sp. nov. urn:1sid:zoobank.org:act:60F5152B-4228-40B2-B1C7-E8EE3A4E6C39}

Figs 7-9; Table 2

\section{Diagnosis}

Transverse bands on Th. III, posterior margin of Abd. II, and Abd. III; cephalic mac $\mathrm{S}_{0}$ and $\mathrm{S}_{0}$ ' present; Th. II with 1 (m1) medio-medial and 12 (p1-6, p1i, p2a, p2p, p2a, p2e, p3p) posterior mac; chaetae a2 and 33 as mac on Th. III; Abd. I with 3 (m2-4) mac; Abd. III with 2 (a2, m3) central mac; Abd. IV with 5 (A6, B4-6 and one of unclear homology) central mac.

\section{Etymology}

Named after the great similarities between the new species and W. buskii (Lubbock, 1869).

\section{Material examined}

\section{Holotype}

CHINA: ${ }^{\top}$ on slide, Anhui Province, Liuan, Jinzhai County, Tiantangzhai, $31.188^{\circ} \mathrm{N}, 115.802^{\circ} \mathrm{E}$, altitude 564 m, 2 Aug. 2012, Z.X. Pan and Q.P. Ren leg. (slide \# 4368_1, sample \# 4368), deposited in NJAU.

\section{Paratypes}

CHINA: $4+q$ on slides and 2 in alcohol, same data as holotype. One paratype on slide (slide \# 4368_5) deposited in MNHN, 2 in alcohol deposited in TZU and others (slide \# 4368_2-4) in NJAU.

\section{Description}

Body length up to $1.50 \mathrm{~mm}$.

Ground colour pale in alcohol. Ant. I-IV gradually darker. A transverse band between the eye patches. Dark stripe on lateral margin of Th. II-Abd. IV. Transverse bands on Th. III, posterior margin of Abd. II, and Abd. III. Irregular longitudinal stripes on lateral part of Abd. IV. Abd. V and Abd. IV posteriorly dark. Base of legs, distal femora, and tibiotarsi pigmented (Fig. 7A-C). Scales brown, pointed, heavily striate with basal ribs longer than distal ones, and only present dorsally on head and terga. Partial chaetae of the row along the posterior margin of each tergum transformed into scales and larger than other scales (Fig. 7E-H).

Based on 7 measured specimens, antenna 2.22-2.53 times as long as cephalic diagonal. Antennal segment ratio as I: II: III: IV = 1: $1.68-2.20: 1.88-2.25: 2.81-3.23$. Smooth spiny mic at base of antennae: 3 dorsal and 4 ventral on Ant. I; 1 internal, 1 external and 2 ventral on Ant. II.

Labral intrusion U-shaped. Clypeal chaetae 10 in number, all ciliate and 5 of them smaller (Fig. 8B). Dorsal cephalic chaetotaxy with 4 antennal (An), 4 median (M) and 8 sutural (S) mac; mac $\mathrm{S}_{1}$ absent; $\mathrm{S}_{0}$ and $\mathrm{S}_{0}{ }^{\prime}$ as mac on middle line; $\mathrm{A}_{0}$ as mic in one specimen (Fig. $8 \mathrm{C}$ ); interocular chaeta $\mathrm{r}$ absent on one side in one specimen. Tip of lateral process of labial palp just reaching apex of labial papilla (Fig. 8E). Labial chaeta $\mathrm{R} / \mathrm{M}=0.58-0.70$; chaeta $\mathrm{R}$ as a scale on one side in one specimen. Cephalic groove with $7+7(8)$ ciliate chaetae (Fig. 8F).

Metatrochanteral organ with 10-17 smooth spine-like chaetae; 8-12 in L-shaped arms and 2-5 between arms (Fig. 8G). Ungual basal paired inner teeth with tip reaching 55\% from base, median one at $75 \%$ and distal one at $87 \%$. Tenent hairs subequal to unguis in length (Fig. 8H). Abd. IV 2.76-3.55 times as long as Abd. III along dorsal midline. Ventral tube anteriorly with about 13 ciliate chaetae, 3 of them larger than others (Fig. 8I); posteriorly with 7-11 chaetae and most of them weakly ciliate except most apical two (Fig. 8J); each lateral flap with 10 chaetae and 3 of them ciliate (Fig. 8K). Male genital plate 

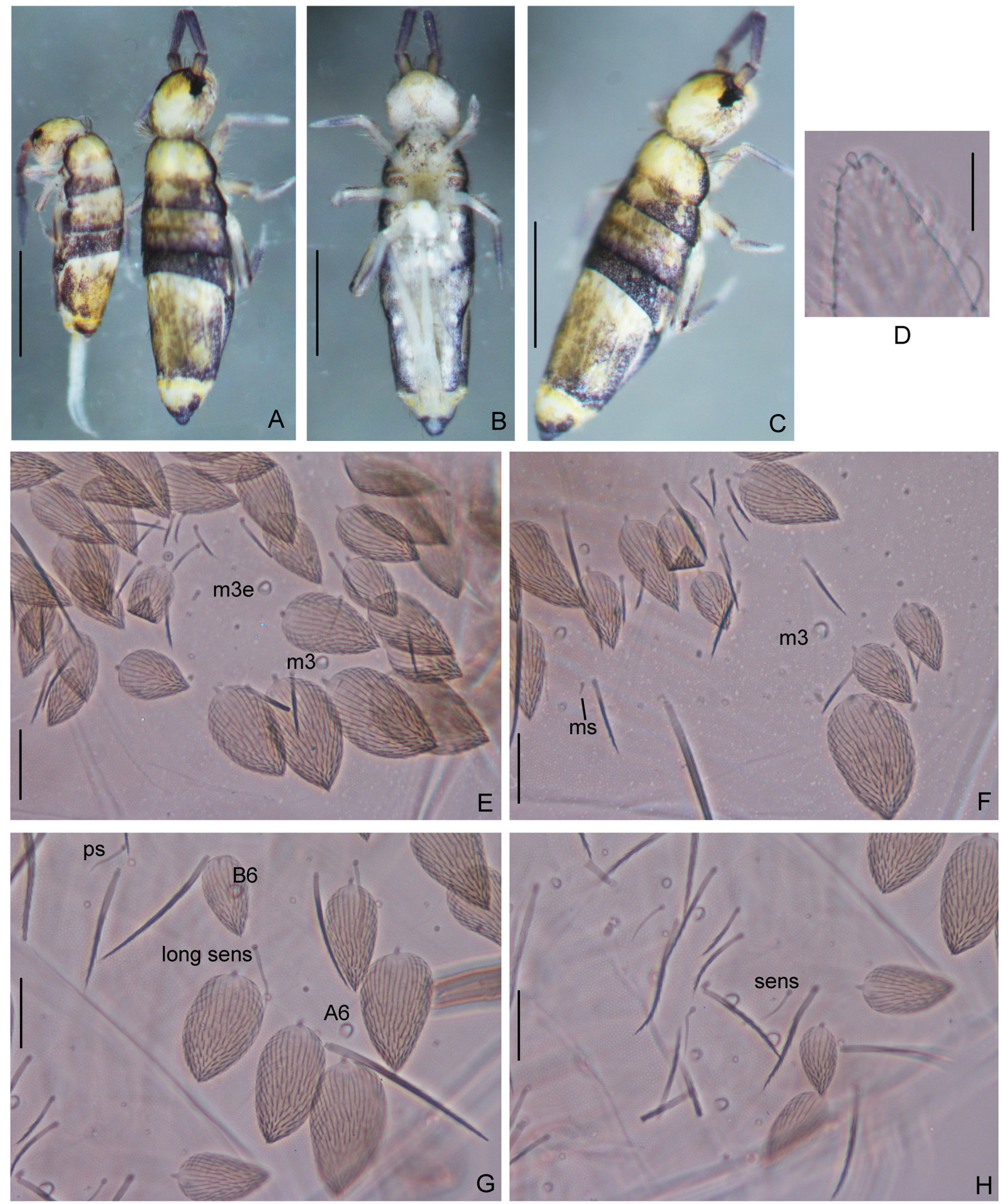

Fig. 7. Willowsia pseudobuskii sp. nov. A-C. Habitus, A and C dorsal view, B ventral view. D. Ant. IV apical bulb. E-H. Body scales. E. Abd. II. F. Abd. III. G. Abd. IV. H. Abd. V. Scale bars: A-C = $500 \mu \mathrm{m} ; \mathrm{D}-\mathrm{H}=20 \mu \mathrm{m}$. 


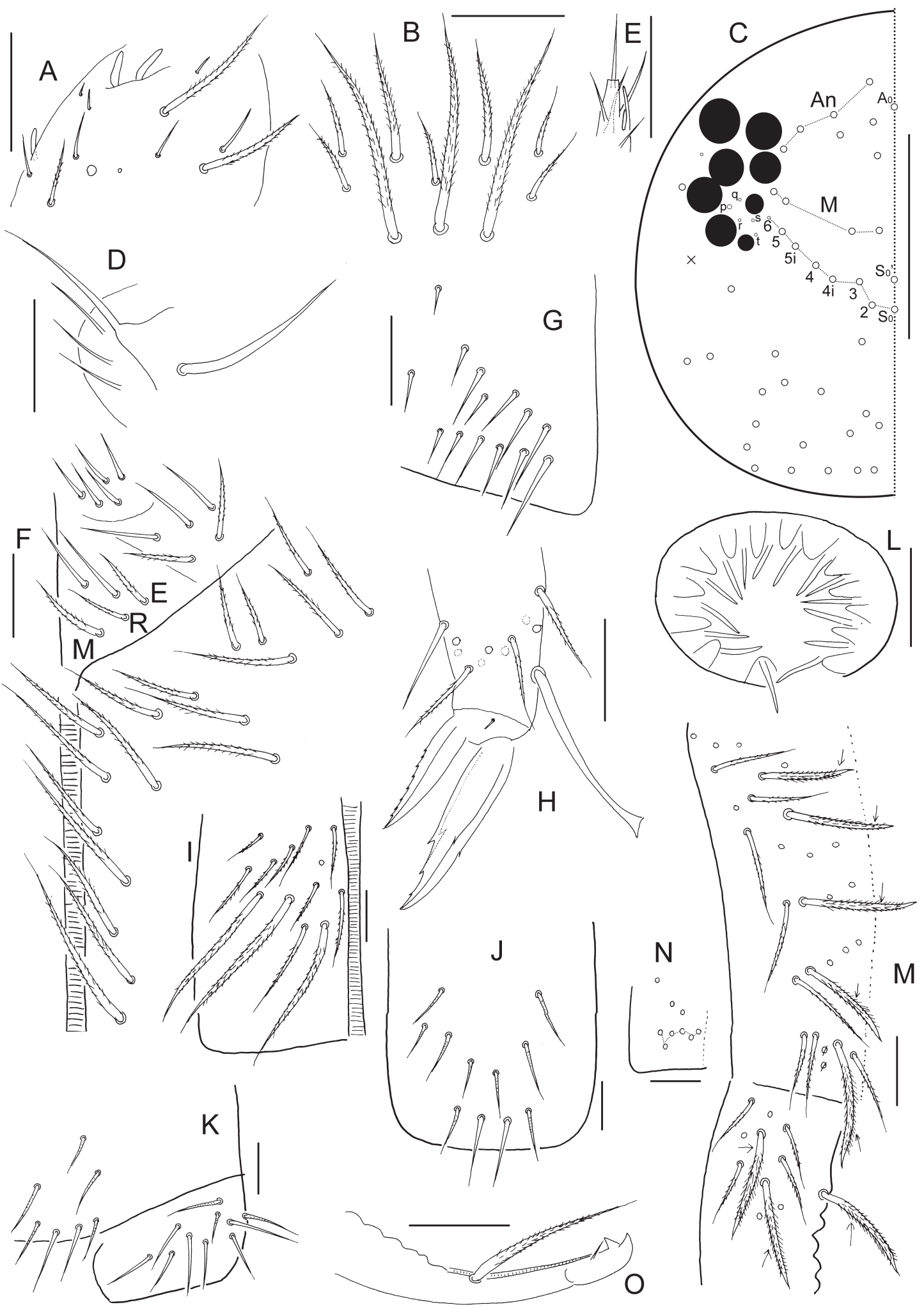

Fig. 8. Willowsia pseudobuskii sp. nov. A. Ant. III organ. B. Clypeal chaetae. C. Dorsal cephalic chaetotaxy. D. Maxillary outer lobe. E. Lateral process of labial palp. F. Chaetae on ventral side of head. G. Trochanteral organ. H. Hind claw. I-K. Ventral tube. I. Anterior face. J. Posterior face. K. Lateral flap. L. Male genital plate. M. Manubrium and base of dens, dorsal view. N. Distal part of ventral face of manubrium. O. Mucro. Scale bars: A-B, D-O $=20 \mu \mathrm{m} ; \mathrm{C}=100 \mu \mathrm{m}$. 
papillate with 15 projections (Fig. 8L). Manubrium and base of dens dorsally with 5-6 and 3 large differentiated chaetae, respectively (Fig. 8M); ventrally with 6+6 distal chaetae (Fig. 8N). Manubrial plaque with 2 pseudopores and 4 ciliate chaetae (Fig. 8M). Distal smooth part of dens 1.46-1.82 times as long as mucro.

Th. II with $1(\mathrm{~m} 1)$ medio-medial, $2(\mathrm{~m} 4, \mathrm{~m} 4 \mathrm{i})$ medio-lateral, and 12 posterior mac; $\mathrm{m} 5$ and $\mathrm{p} 6$ as mac. Th. III with 15 mac; a1 as mic (Fig. 9A). Abd. I with 3 (m2-4) mac. Abd. II with 3 (a2, m3, m3e) central
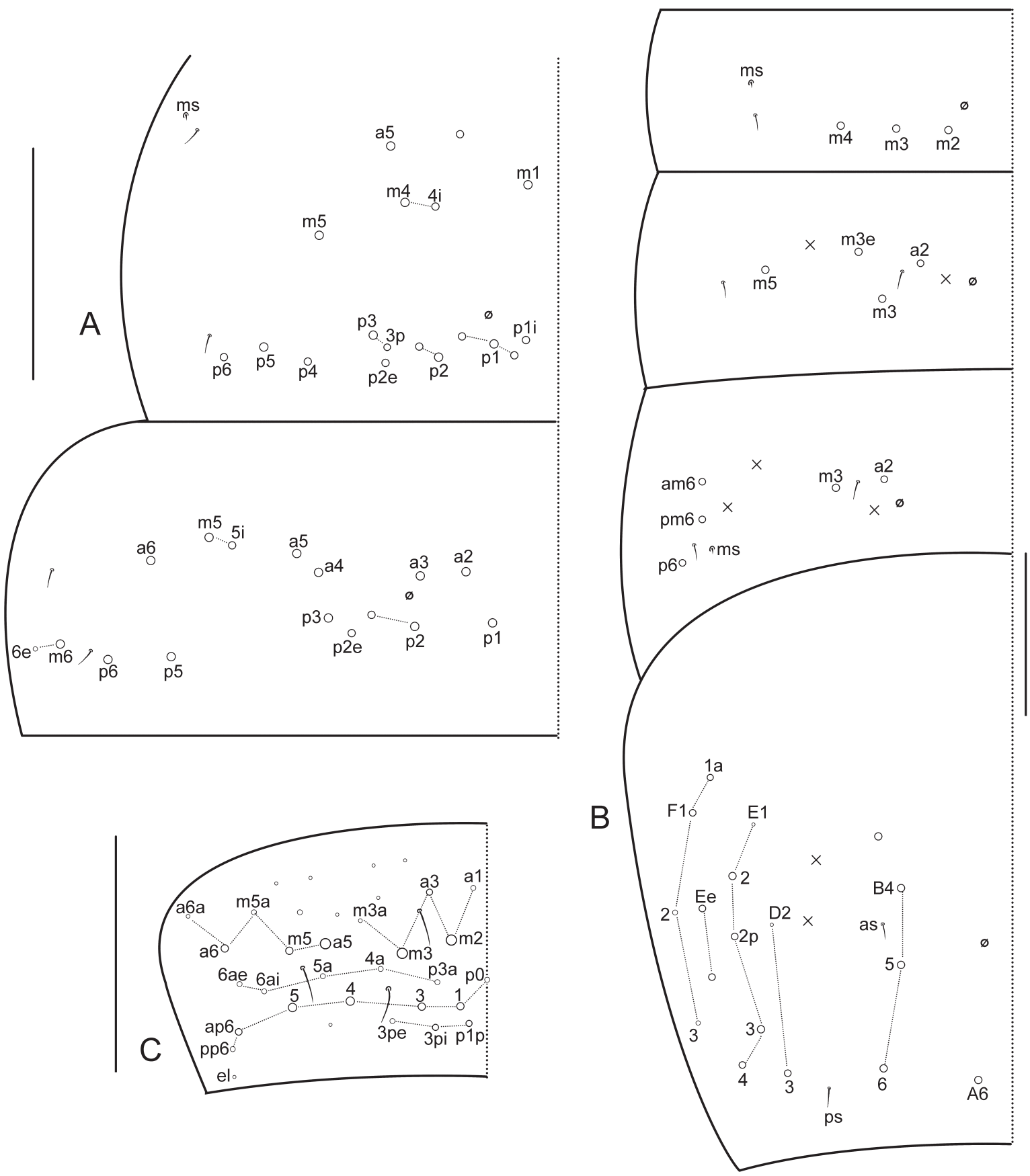

Fig. 9. Willowsia pseudobuskii sp. nov. A. Thoracic chaetotaxy. B-C. Abdominal chaetotaxy. B. Abd. I-IV. C. Abd. V. Scale bars: $100 \mu \mathrm{m}$. 
PAN Z. \& ZHANG F., Willowsia having scales of the long rib type

Table 2. Comparison between W. pseudobuskii sp. nov. and W. buskii (Lubbock, 1869).

\begin{tabular}{lcc}
\hline Characters & $\begin{array}{c}\text { W. pseudobuskii } \\
\text { sp. nov. }\end{array}$ & $\begin{array}{c}\text { W. buskii } \\
\text { (Lubbock, 1869) }\end{array}$ \\
\hline Pigment dorsally on body & forming a pattern & scattered \\
$\mathrm{S}_{0}{ }^{\prime}$ dorsally on head & present & absent \\
Chaetae m1, m5, p1i, p2e and p6 on Th. II & mac & mic \\
Chaetae p4i and p5pi on Th. II & mic & mac \\
Chaetae a2, a3 and m5i on Th. III & mac & mic \\
\hline
\end{tabular}

and 1 (m5) lateral mac. Abd. III with 2 (a2, m3) central and 3 (am6, pm6, p6) lateral mac. Abd. IV with 5 central and about 9 lateral mac (Fig. 9B).

\section{Ecology}

In decomposing litter of Oryza sativa Linnaeus, 1753.

\section{DNA barcodes}

KU833223, KU833224.

\section{Remarks}

Willowsia pseudobuskii sp. nov. is characterized by the unique colour pattern and the dorsal chaetotaxy of the head and Abd. IV. The new species is closest to W. buskii in many features, particularly the chaetotaxy of Abd. II-IV. However, if differs from the latter in colour pattern, number of sutural chaetae, and thoracic chaetotaxy (Table 2). The genetic distances of COI between the new species and W. buskii (five COI sequences from GenBank, LK024534-LK024536, HG422601, KM610129) are 0.178-0.191, indicating two independent species.

\section{Willowsia similis sp. nov. urn:Isid:zoobank.org:act:427FEB52-E400-490E-89C7-72CB02AE9EE6}

Figs 10-12; Table 1

\section{Diagnosis}

Transverse bands on Th. III and Abd. II-III; cephalic mac $\mathrm{S}_{0}$ absent; Th. II with 1 (m1) medio-medial and $2(\mathrm{~m} 4, \mathrm{~m} 4 \mathrm{i})$ medio-lateral, and $12(\mathrm{p} 1-6, \mathrm{p} 1 \mathrm{i}, \mathrm{p} 2 \mathrm{a}, \mathrm{p} 2 \mathrm{p}, \mathrm{p} 2 \mathrm{a}, \mathrm{p} 2 \mathrm{e}, \mathrm{p} 3 \mathrm{p})$ posterior mac; chaetae a1 and a3 on Th. III as mic; Abd. I with 3 (m2-4) mac; Abd. III with 3 (a2, a3, m3) central mac.

\section{Etymology}

Named after the similarity to Willowsia fascia Zhang \& Pan sp. nov.

\section{Material examined}

\section{Holotype}

CHINA: + on slide, Hunan Province, Changsha City, Yuelu Mountain, $28.195^{\circ} \mathrm{N}, 112.944^{\circ} \mathrm{E}$, altitude 159 m, 9 Aug. 2012, Z.X. Pan and Q.P. Ren leg. (slide \# 4381_1, sample \# 4381), deposited in NJAU.

\section{Paratypes}

CHINA: 3 우 on slides and 3 in alcohol, same data as holotype. One paratype on slide (slide \# 4381_4) deposited in MNHN, 3 in alcohol in TZU, and others (4381_2-3) in NJAU 


\section{Description}

Body length up to $1.83 \mathrm{~mm}$.

Ground colour yellow in alcohol. Ant. I-IV gradually darker. Transverse bands on Th. III and Abd. II-III (Fig. 10A-B). Scales brown, pointed, heavily striate with basal ribs very long, and only present dorsally on head and terga. Partial chaetae of the row along the posterior margin of each tergum transformed into scales and larger than other scales (Fig. 10D).

Based on 7 measured specimens, antenna 1.95-2.44 times as long as cephalic diagonal. Antennal segment ratio as I: II: III: IV = 1: 2.07-2.30: 2.07-2.30:2.45-2.93. Smooth spiny mic at base of antennae: 3 dorsal and 3 ventral on Ant. I; 1 internal, 1 external and 1 ventral on Ant. II.

Labral intrusion U-shaped. Clypeal chaetae 10 in number, all ciliate and 3 of them smaller. Dorsal cephalic chaetotaxy with 4 antennal (An), 4 median (M) and 6 sutural (S) mac; mac $\mathrm{S}_{0}, \mathrm{~S}_{1}$ and $\mathrm{Ps}_{2}$ absent (Fig. 11B). Tip of lateral process of labial palp not reaching apex of labial papilla. Labial chaeta $\mathrm{R} / \mathrm{M}=$ 0.62-0.71. Cephalic groove with $6+6$ ciliate chaetae (Fig. 11C).

Metatrochanteral organ with 10-17 smooth spine-like chaetae; 8-12 in L-shaped arms and 2-5 between arms (Fig. 11D). Ungual basal paired inner teeth with tip reaching 51\% from base, median one at $73 \%$ and distal one at $88 \%$. Tenent hairs subequal to unguis in length (Fig. 11E). Abd. IV 3.21-3.45 times as long as Abd. III along dorsal midline. Ventral tube anteriorly with at least $11+11$ ciliate chaetae (Fig. 11F); posteriorly not clearly seen; each lateral flap with about 9 chaetae and 3 of them weakly ciliate. Manubrium and base of dens dorsally with about 12 and 3 large differentiated chaetae, respectively (Fig. 11G); ventrally with $6+6$ distal chaetae (Fig. 11H). Manubrial plaque with 2 pseudopores and 4 ciliate chaetae (Fig. 11G). Distal smooth part 1.81 times as long as mucro.

Th. II with 1 (m1) medio-medial, 2 (m4, m4i) medio-lateral, and 12 posterior mac; $\mathrm{p} 6$ as mac. Th. III with 14 mac; a1 and a3 as mic (Fig. 12A). Abd. I with 3 (m2-4) mac. Abd. II with 3 (a2, m3, m3e) central
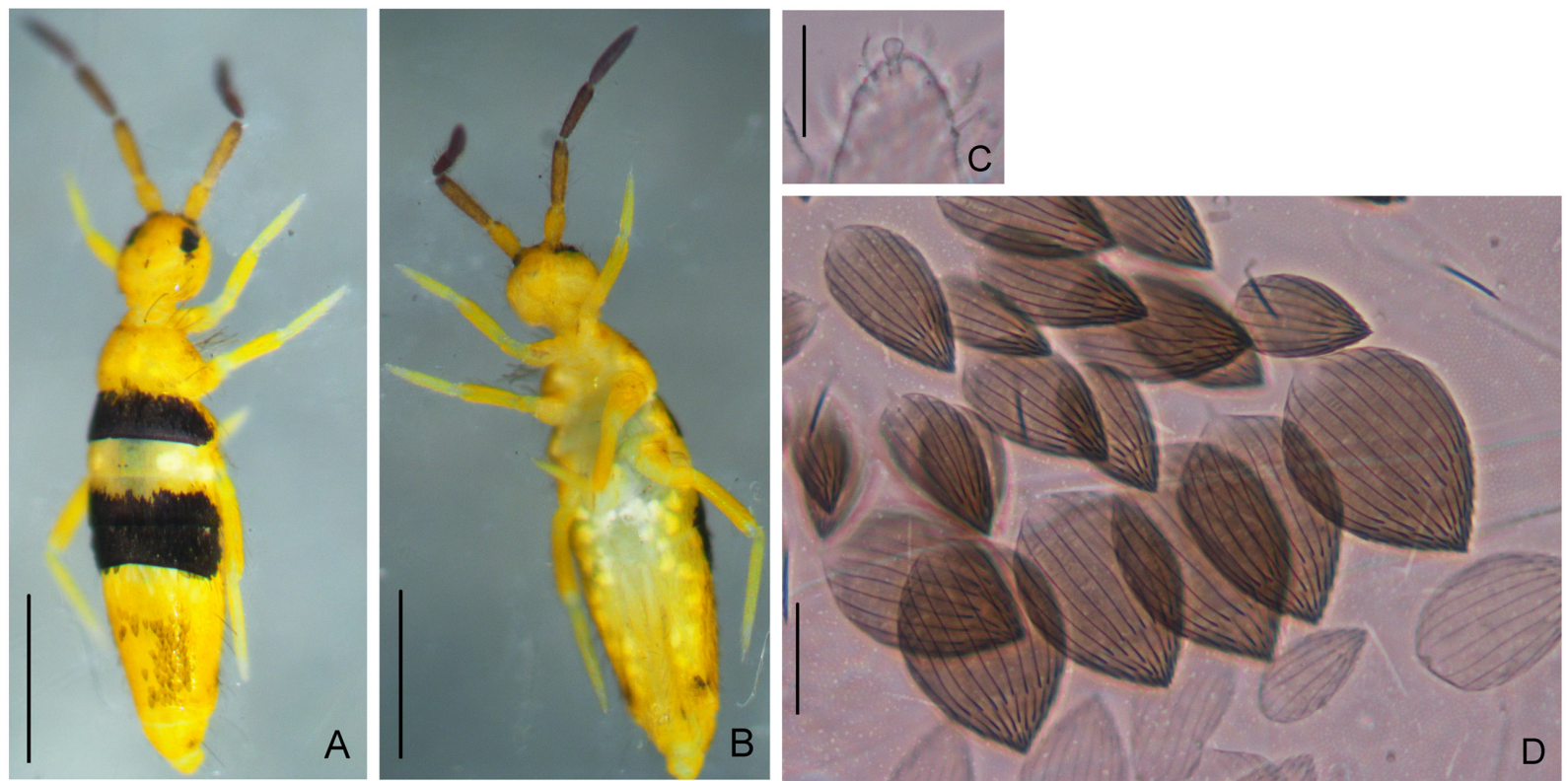

Fig. 10. Willowsia similis sp. nov. A-B. Colour pattern. A. Dorsal view. B. Ventral view. C. Ant. IV apical bulb. D. Scales on Th. III. Scale bars: A-B $=500 \mu \mathrm{m} ; \mathrm{C}-\mathrm{D}=20 \mu \mathrm{m}$. 


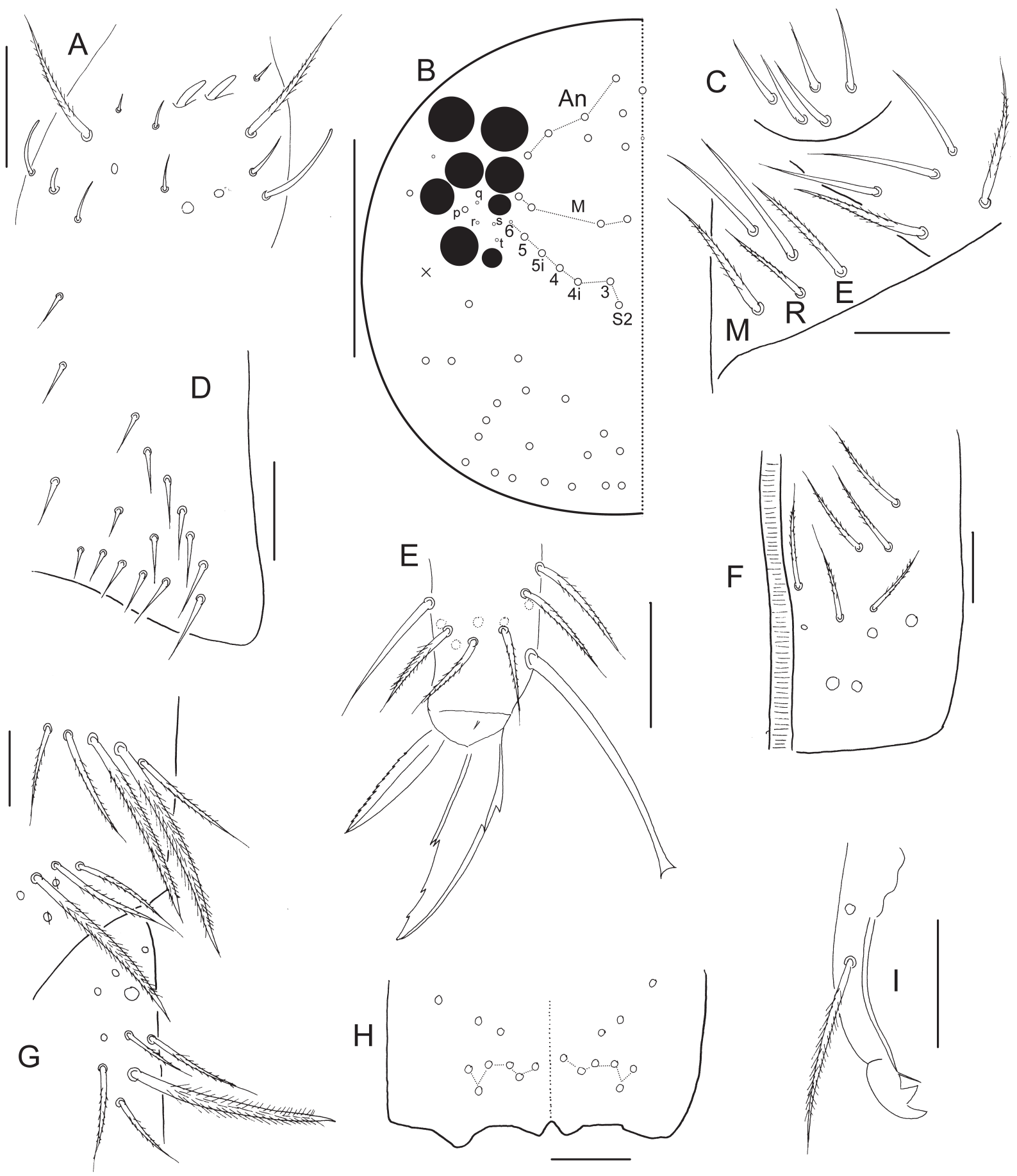

Fig. 11. Willowsia similis sp. nov. A. Ant. III organ. B. Dorsal cephalic chaetotaxy. C. Labium. D. Trochanteral organ. E. Hind claw. F. Anterior face of ventral tube. G. Manubrium and base of dens, dorsal view. H. Distal parts of ventral face of manubrium. I. Mucro. Scale bars: A, C-I $=20 \mu \mathrm{m}$; B = $100 \mu \mathrm{m}$. 
and 1 (m5) lateral mac. Abd. III with 3 (a2, a3, m3) central and 3 (am6, pm6, p6) lateral mac. Abd. IV with 7 central and 10 lateral mac; F2 and F3 as mesochaetae (Fig. 12B).

\section{Ecology}

In decomposing leaves of Cinnamomum camphora (L.) Nees \& Eberm. in the forest.

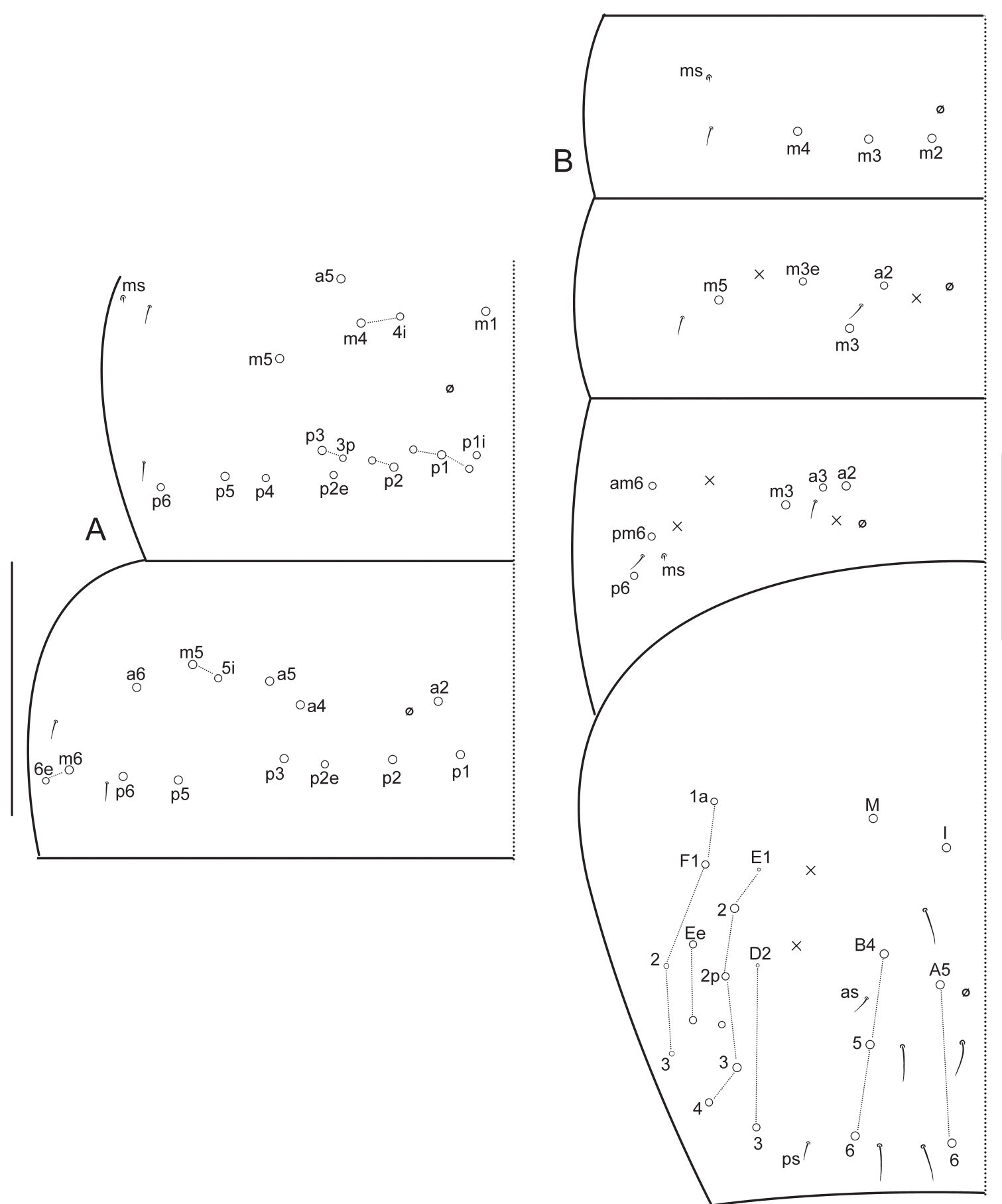

Fig. 12. Willowsia similis sp. nov. A. Thoracic chaetotaxy. B. Chaetotaxy. of Abd. I-IV (long sens on Abd. IV incomplete). Scale bars: $100 \mu \mathrm{m}$. 


\section{DNA barcode}

\section{KU833225.}

\section{Remarks}

Willowsia similis sp. nov. is characterized by the transverse bands on Th. III and Abd. II-III. For more comments, see remarks above on W. fascia Zhang \& Pan sp. nov.

Willowsia qui Zhang, Chen \& Deharveng, 2011

Figs 13-15; Table 3

\section{Diagnosis}

Very narrow lateral stripes on Th. II-Abd. I and transverse bands on postero-lateral margin of Abd. II-III; cephalic mac $\mathrm{S}_{0}$ absent but $\mathrm{S}_{0}$ present; 7 clypeal chaetae; Th. II with 2 medio-medial, 3 mediolateral, and 12-13 posterior mac; chaeta al as mic on Th. III; Abd. I with 4 mac; Abd. III with 2 central mac; Abd. IV with 6 central mac.

\section{Material examined}

CHINA: holotype and paratype on slides, Zhejiang Province, Taishun County, Wuyanling National Forest Park, Shuangkengkou, $27.617^{\circ}$ N, $119.767^{\circ}$ E, altitude 600 m, 4 Aug. 2005, J.X. Chen team leg.

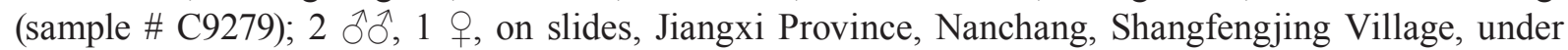
leaf litter of bamboo, $28.792^{\circ} \mathrm{N}, 115.808^{\circ} \mathrm{E}$, altitude $118 \mathrm{~m}, 11$ Aug. 2012, Z.X. Pan and Q.P. Ren leg.

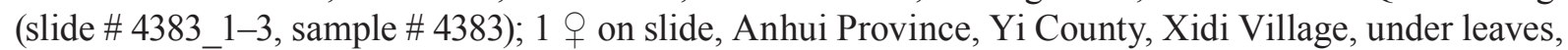
$29.905^{\circ} \mathrm{N}, 1 \overline{17.992^{\circ}} \mathrm{E}$, altitude $223 \mathrm{~m}, 18$ Nov. 2012, F. Zhang, Z.-X. Pan and Z.-H. Li leg. (slide \# HC13_1, sample \# HC13); 1 q on slide, Anhui Province, Tangkou Town, Fuxi Village, bamboo forest, $30.08^{\circ} \mathrm{N}, 118.148^{\circ} \mathrm{E}$, altitude $689 \mathrm{~m}, 15 \mathrm{Jul} .2015$, F. Zhang leg. (slide \# 15AH10, sample \# 15AH_ monkey).

\section{Description}

Body length up to $1.8 \mathrm{~mm}$.

Ground colour pale in alcohol. Ant. I-II with distal patches and III-IV dark. A transverse band between eye patches. Narrow stripes present on lateral margin of Th. II-Abd. I. Narrow transverse bands on posterior margin of Abd. II-III. Small patches posteriorly on Abd. IV-VI (Fig. 13A). Scales pointed, heavily striate with basal ribs longer than distal ones, and only present dorsally on head and terga. Some chaetae of the row along the posterior margin of each tergum transformed into scales and larger than other scales (Fig. 13B).

Based on 5 measured specimens, antenna 2.48-2.54 times as long as cephalic diagonal. Antennal segment ratio as I: II: III: IV = 1: $1.69-2.12: 1.69-2.00: 2.65-3.15$. Smooth spiny mic at base of antennae: 3 dorsal and 2 ventral on Ant. I; 1 internal, 1 external and 1 ventral on Ant. II.

Labral intrusion slightly curved. Seven clypeal chaetae ciliate and arranged in two rows (Fig. 14B). Dorsal cephalic chaetotaxy with 4 antennal (An), 4 median (M) and 7 sutural (S) mac; mac $\mathrm{S}_{0}{ }^{\prime}$ present; mac $S_{0}$ and $S_{1}$ absent; mac $P_{2}$ rarely absent (Fig. 14C). Tip of lateral process of labial palp slightly beyond apex of labial papilla. Labial chaeta $\mathrm{R} / \mathrm{M}=0.65-0.76$. Cephalic groove with $7(6)$ ciliate chaetae on each side.

Metatrochanteral organ with 10-14 smooth spine-like chaetae; 8-11 in L-shaped arms and 2-3 between arms (Fig. 14D). Ungual basal paired inner teeth with tip reaching $52 \%$ from base, median one at $75 \%$ 
Table 3. Comparison between $W$. qui Zhang, Chen \& Deharveng, 2011 and $W$. japonica Folsom, 1897.

\begin{tabular}{|c|c|c|}
\hline Characters & $\begin{array}{c}\boldsymbol{W} \cdot \boldsymbol{q u i} \\
\text { Zhang, Chen \& Deharveng, } 2011\end{array}$ & $\begin{array}{c}\text { W. japonica } \\
\text { Folsom, } 1897\end{array}$ \\
\hline Stripes dorsally on body & very narrow & normal \\
\hline Small patch dorsally on Th. III & absent & present \\
\hline Scale width & broad & narrow \\
\hline Scale surface sculpture & long basal rib type & short rib type \\
\hline Smooth part of dens/mucro & $1.6-1.8$ & $2.2-2.9$ \\
\hline \multicolumn{3}{|l|}{ Chaetotaxy of Th. II } \\
\hline $\mathrm{m} 1$ and $\mathrm{m} 5$ & $\operatorname{mac}$ & mic \\
\hline $\mathrm{mac} m 4 \mathrm{i}$ and $\mathrm{m} 4 \mathrm{p}$ & present & absent \\
\hline Mac on Abd. I & 4 & 3 \\
\hline Central mac on Abd. IV & 6 (A5 as meso) & 7 (A5 as mac) \\
\hline
\end{tabular}

and distal one at $88 \%$. Tenent hairs slightly longer than unguis (Fig. 14E). Abd. IV 3.47-3.70 times as long as Abd. III along dorsal midline. Ventral tube anteriorly with 12-15 ciliate chaetae, 5-7 of them larger than others (Fig. 14F); posteriorly with 6-8 smooth chaetae; each lateral flap with 11 chaetae (Fig. 14G). Manubrium dorsally with $6+6$ thick chaetae, 3 large and 3 ( 1 in distal plaque) median in size (Fig. 14H); ventrally with $8(7)+8(7)$ distal chaetae (Fig. 14I). Manubrial plaque with 2 pseudopores and 4 ciliate chaetae (Fig. 14H). Distal smooth part 1.60-1.81 times as long as mucro.

Th. II with 2(3) (m1, m2) medio-medial, $3(\mathrm{~m} 4, \mathrm{~m} 4 \mathrm{i}, \mathrm{m} 4 \mathrm{p})$ medio-lateral and 12-13 posterior mac; mac $\mathrm{m} 2 \mathrm{i}$ present in one specimen; $\mathrm{m} 5$ and $\mathrm{p} 4-6$ as mac; mac $\mathrm{p} 2 \mathrm{a}$ often absent. Th. III with 14 mac; al as mic; m6e as mesochaeta (Fig. 15A). Abd. I with 4 (m2-4, m4p) mac. Abd. II with 3 (a2, m3, m3e) central and 1 (m5) lateral mac. Abd. III with 2 (a2, m3) central and 3 (am6, pm6, p6) lateral mac; a3 as mac in one specimen. Abd. IV with 6 central mac, about 12 lateral mac and at least 9 sens; A5 as mesochaeta (Fig. 15B).
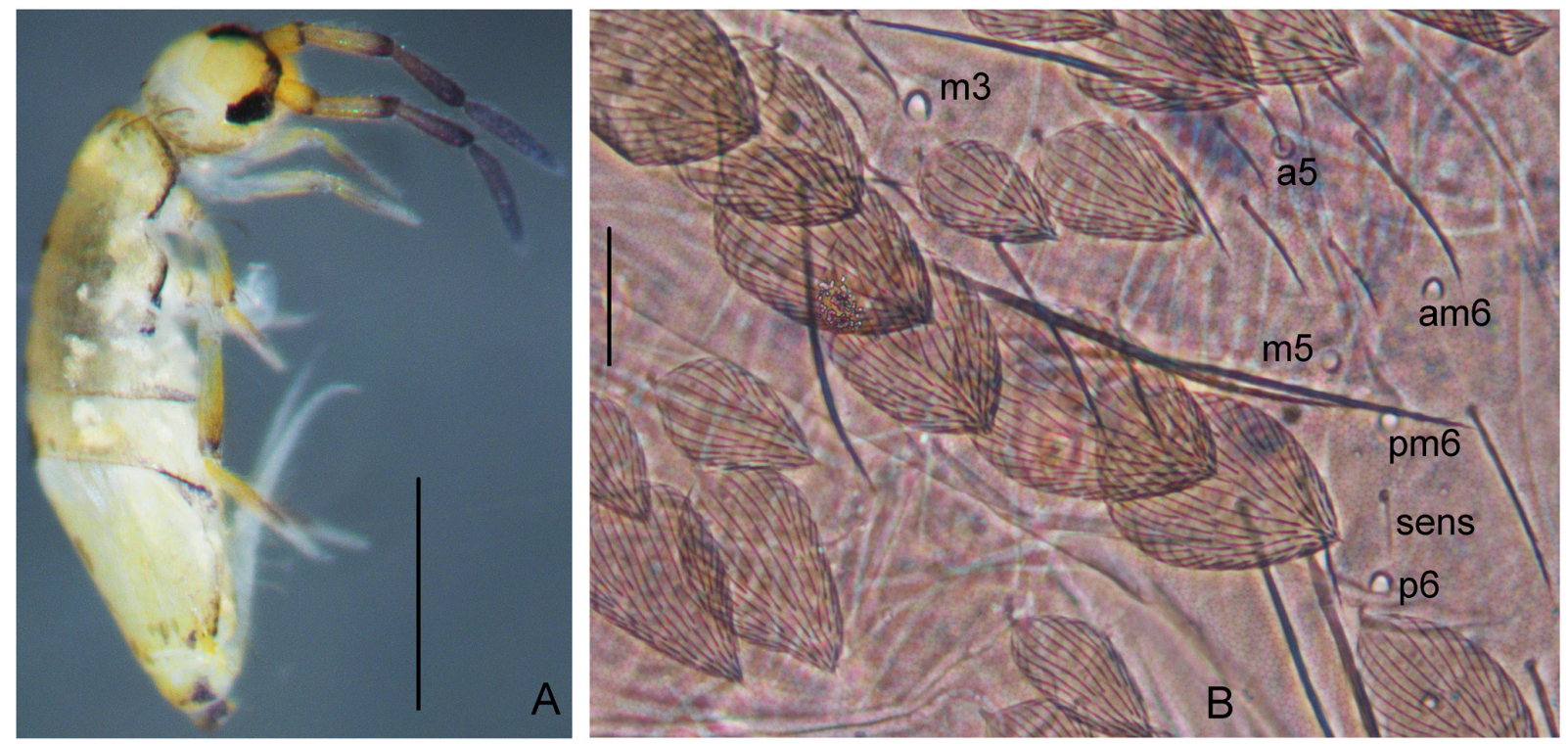

Fig. 13. Willowsia qui Zhang, Chen \& Deharveng, 2011. A. Habitus. B. Abd. III. Scale bars: A = $500 \mu \mathrm{m}$; $\mathrm{B}=20 \mu \mathrm{m}$. 

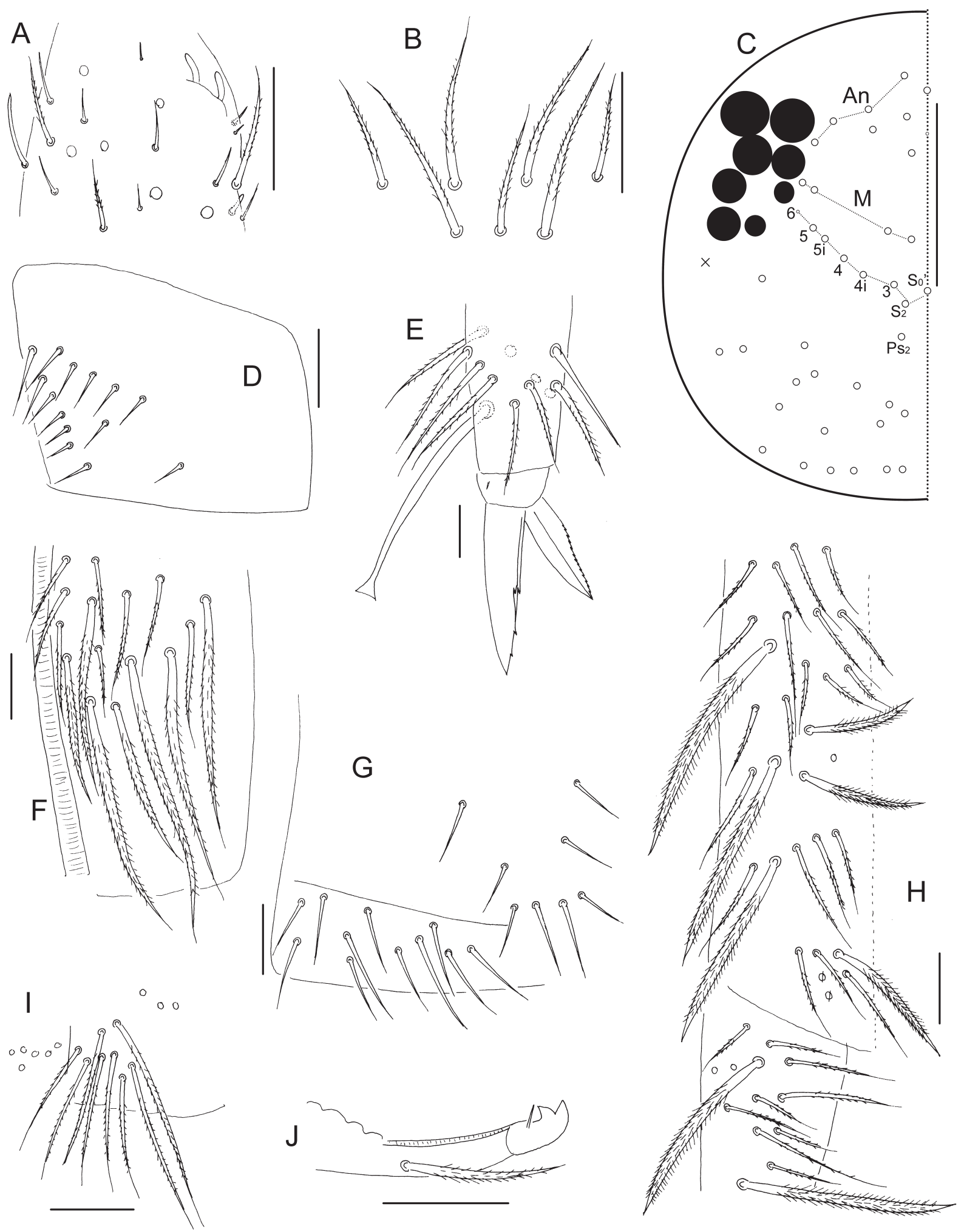

Fig. 14. Willowsia qui Zhang, Chen \& Deharveng, 2011. A. Ant. III organ. B. Clypeal chaetae. C. Dorsal cephalic chaetotaxy. D. Trochanteral organ. E. Hind claw. F. Anterior face of ventral tube. G. Posterior face and lateral flap of ventral tube. H. Manubrium and base of dens, dorsal view. I. Distal chaetae of ventral face of manubrium. J. Mucro. Scale bars: A-B, D-J $=20 \mu \mathrm{m} ; \mathrm{C}=100 \mu \mathrm{m}$. 


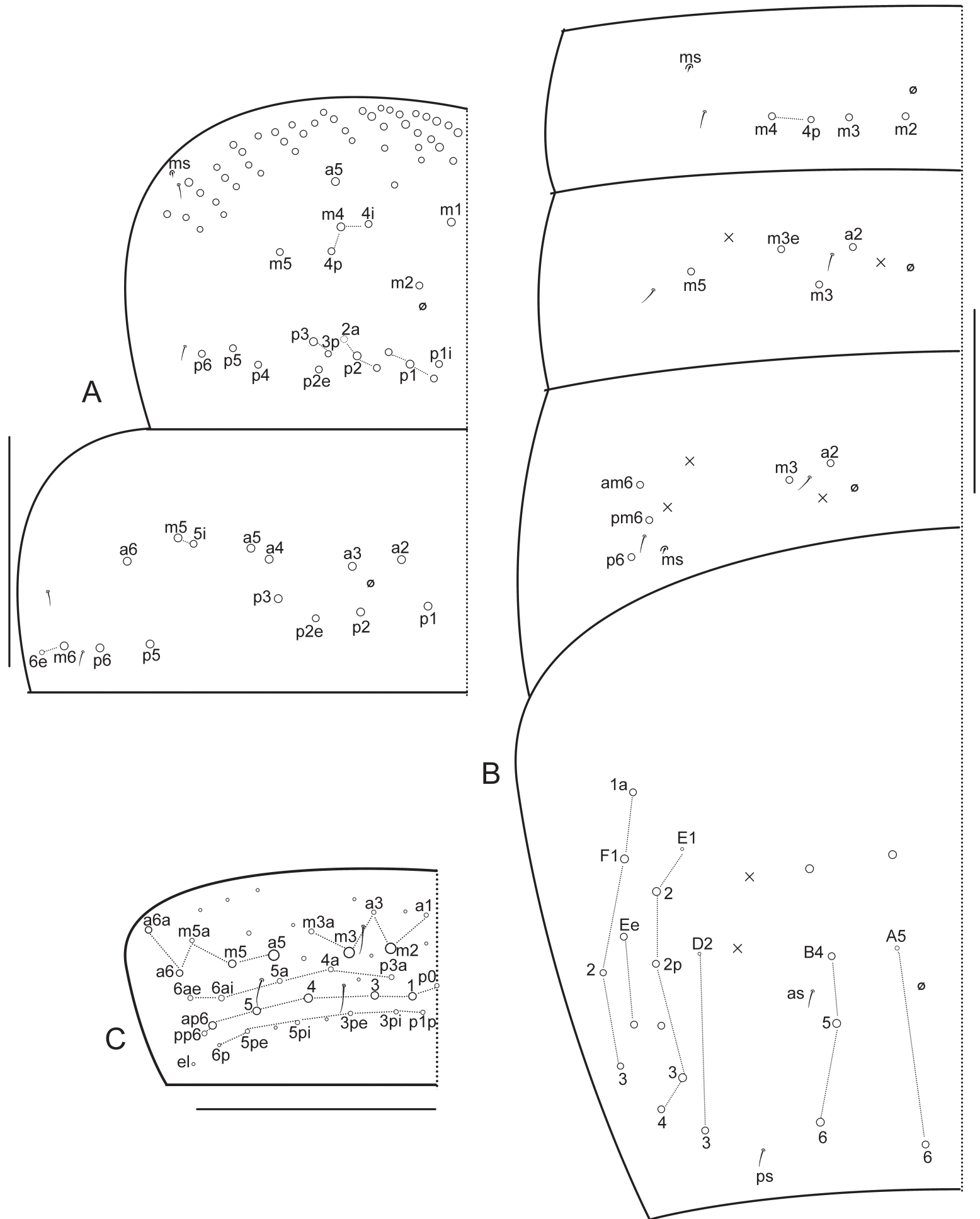

Fig. 15. Willowsia qui Zhang, Chen \& Deharveng, 2011. A. Thoracic chaetotaxy. B-C. Abdominal chaetotaxy. B. Abd. I-IV. C. Abd. V. Scale bars: $100 \mu \mathrm{m}$. 


\section{Ecology}

Found in litter, debris and rotten logs of evergreen broad-leaved forest.

\section{DNA barcodes}

KU833221, KU833226.

\section{Remarks}

We found a paratype, which was actually $W$. japonica (Folsom, 1897) and incorrectly designated as $W$. $q u i$ by the original authors, resulting in some mistakes in the original description. Based on the types and fresh material, $W$. qui differs from $W$. japonica in narrower stripes on lateral margin of thorax and posterior margin of Abd. II-III, broad body scales, short distal smooth part of dens, and chaetotaxy of Th. II and Abd. I and IV (Table 3). Mesochaeta A5 is as long as macrochaetae, but thinner, with small socket and pointed tip. The K2P genetic distance (0.184-0.192) of the COI (one sequence of $W$. japonica from GenBank: KM978378) also indicates two independent species.

\section{Key to the Willowsia species having scales of the long rib type}

1. Mucro with subapical tooth much smaller than apical one and basal spine long, reaching midway between the subapical and apical teeth W. samarkandica Martynova, 1972

- Mucro with teeth subequal and basal spine short, reaching the apex of subapical tooth 2

2. Unguiculus truncate W. formosana (Denis, 1929)

- Unguiculus lanceolate 3

3. Pigment scattered on all terga W. buskii (Lubbock, 1869)

- Pigment not uniformly distributed on each tergum 4

4. Ant. I-II and ventral side of manubrium scaled ........W. guangdongensis Zhang, Xu \& Chen, 2007

- Antennae and manubrium unscaled

5. Each labral papilla with at least two denticles W. jacobsoni (Börner, 1913)

- Labral papillae conical

6. Abd. I with $7+7$ mac; Abd. II with $6+6$ central mac ...W. potapovi Zhang, Chen \& Deharveng, 2011

- Abd. I with at most $4+4$ mac; Abd. II with at most $5+5$ central mac

7. Abd. II with $5+5$ central mac W. yiningensis Zhang, Chen \& Deharveng, 2011

- Abd. II with at most $4+4$ central mac 8

8. Abd. II with $4+4$ central mac W. mekila Christiansen \& Bellinger, 1992

- Abd. II with $3+3$ central mac

9. Abd. I with $4+4$ mac W. qui Zhang, Chen \& Deharveng, 2011

- Abd. I with at most 3+3 mac

10. Abd. III with $2+2$ central mac

- Abd. III with $3+3$ central mac

11. Cephalic mac $\mathrm{S}_{0}$ and $\mathrm{S}_{0}{ }^{\prime}$ present; Abd. IV with $5+5$ central mac W. pseudobuskii sp. nov.

- Cephalic mac $\mathrm{S}_{0}$ and $\mathrm{S}_{0}{ }^{\prime}$ absent; Abd. IV with $7+7$ central mac W. pseudoplatani Zhang \& Pan sp. nov. 
12. Abd. II-III with transverse bands

- Abd. II-III without transverse bands W. nigromaculata (Lubbock, 1873)

13. Metathorax pigmented; cephalic $\mathrm{S}_{0}$ absent; $\mathrm{a} 3$ on Th. III as mic

- Metathorax unpigmented; cephalic $\mathrm{S}_{0}$ present; $\mathrm{a} 3$ on Th. III as mac

.W. fascia Zhang \& Pan sp. nov.

14. Abd. IV unpigmented; Th. II with $1+1$ medio-medial and $2+2$ medio-lateral mac

W. similis sp. nov.

- Abd. IV pigmented; Th. II with only 1+1 medio-lateral mac W. platani (Nicolet, 1842)

\section{Discussion}

Willowsia has the most diversified scale morphology (shape and sculpture) in Collembola, with four basic scale types recognized within the genus (Zhang et al. 2011). However, scale morphology, size and distribution across different terga and different areas of a tergum remain largely undocumented. In this study, some of the chaetae of the row along the posterior margin of each tergum were found to be transformed into scales and larger than other scales, differing from all scales along posterior margin in Lepidocyrtinae and Seirinae. Further examination confirmed that this phenomenon also occurred in many species with scales of the long basal rib type, the short rib type, and the uninterrupted type, although information for some species cannot be traced. In scale distribution, the species with scales of the long basal rib type and the uninterrupted type usually have no scales on the antennae and manubrium (Willowsia guangdongensis Zhang, Xu \& Chen and Willowsia mekila Christiansen \& Bellinger, 1922 excluded). The above evidence implies scale types are likely of high phylogenetic significance within Willowsia.

Within the species having scales of the long basal rib type, W. samarkandica Martynova, 1972, W. jacobsoni (Börner, 1913) and W. guangdongensis seem to be distant from other species. The former two have labral papillae with multiple denticles rather than one. The small subapical tooth and long basal spine on the mucro in $W$. samarkandica are unique. Most species of the group have 7+7 (A5 as mesochaeta in W. qui) central mac on Abd. IV, whereas they are numerous in W. samarkandica and 5+5 in $W$. jacobsoni. W. guangdongensis is the only species having scales on the antennae, legs and ventral side of manubrium. The colour pattern and dorsal chaetotaxy are the main diagnostic characters within the group. Among closely related species, the genetic distances of COI barcodes could provide powerful evidence for species delimitation.

\section{Acknowledgements}

This study was supported by the Zhejiang Provincial Natural Science Foundation of China (LQ14C040002), the Fundamental Research Funds for the Central Universities and a project funded by the Priority Academic Program Development of Jiangsu Higher Education Institutions.

\section{References}

Bellinger P.F., Christiansen K.A. \& Janssens F. 1996-2016. Checklist of the Collembola of the World [online]. Available from http://www.collembola.org [accessed 15 Mar. 2016]

Gisin H. 1967. Espèces nouvelles et lignées évolutives de Pseudosinella endogés (Collembola). Memórias e Estudos do Museu Zoológico da Universidade de Coimbra 301: 1-25.

Jordana R. \& Baquero E. 2005. A proposal of characters for taxonomic identification of Entomobrya species (Collembola, Entomobryomorpha), with description of a new species. Abhandlungen und Berichte des Naturkundemuseums Goerlitz 76: 117-134. 
Mari-Mutt J.A. 1986. Puerto Rican species of Lepidocyrtus and Pseudosinella (Collembola: Entomobryidae). Caribbean Journal of Science 22: 1-48.

Szeptycki A. 1979. Morpho-Systematic Studies on Collembola. IV. Chaetotaxy of the Entomobryidae and its Phylogenetical Significance. Polska Akademia Nauk, Zakład Zoologii Systematycznej i Doświadczalnej, Państwowe Wydawnictwo Naukowe, Warsaw, Krakau.

Tamura K., Peterson D., Peterson N., Stecher G., Nei M. \& Kumar S. 2011. MEGA5: Molecular evolutionary genetics analysis using maximum likelihood, evolutionary distance, and maximum parsimony methods. Molecular Biology and Evolution 28: 2731-2739. http://dx.doi.org/10.1093/molbev/ $\underline{m s r 121}$

Yoshii R. \& Suhardjono Y.R. 1989. Notes on the collembolan Fauna of Indonesia and its vicinities. I. Miscellaneous notes, with special references to Seirini and Lepidocyrtini. Acta Zoologica Asiae Orientalis 1: 23-90.

Zhang F. \& Deharveng L. 2015. Systematic revision of Entomobryidae (Collembola) by integrating molecular and new morphological evidence. Zoologica Scripta 44: 298-311. http://dx.doi.org/10.1111/ $\underline{\text { zsc. } 12100}$

Zhang F., Chen J.X. \& Deharveng L. 2011. New insight into the systematics of the Willowsia complex (Collembola: Entomobryidae). Annales de la Société Entomologique de France 47: 1-20. http://dx.doi. org/10.1080/00379271.2011.10697692

Zhang F., Chen Z., Dong R.-R., Deharveng L., Stevens M.I., Huang Y.-H. \& Zhu C.-D. 2014a. Molecular phylogeny reveals independent origins of body scales in Entomobryidae (Hexapoda: Collembola). Molecular Phylogenetics and Evolution 70: 231-239. http://dx.doi.org/10.1016/j.ympev.2013.09.024

Zhang F., Bedos A. \& Deharveng L. 2014b. Disjunct distribution of Szeptyckiella gen. nov. from New Caledonia and South China undermines the monophyly of Willowsiini (Collembola: Entomobryidae). Journal of Natural History 48: 1299-1317. http://dx.doi.org/10.1080/00222933.2013.859317

Zhang F., Yu D., Luo Y.Z., Ho S.Y.W., Wang B. \& Zhu C.D. 2014c. Cryptic diversity, diversification and vicariance in two species complexes of Tomocerus (Collembola, Tomoceridae) from China. Zoologica Scripta 43: 393-404. http://dx.doi.org/10.1111/zsc.12056

Zhang F., Sun D.-D., Yu D.-Y., Wang B.-X. 2015. Molecular phylogeny supports S-chaetae as a key character better than jumping organs and body scales in classification of Entomobryoidea (Collembola). Scientific Reports 5: 12471. http://dx.doi.org/10.1038/srep12471

Manuscript received: 22 March 2016

Manuscript accepted: 23 May 2016

Published on: 21 November 2016

Topic editor: Gavin Broad

Desk editors: Jeroen Venderickx and Niko Korenhof

Printed versions of all papers are also deposited in the libraries of the institutes that are members of the EJT consortium: Muséum national d'Histoire naturelle, Paris, France; Botanic Garden Meise, Belgium; Royal Museum for Central Africa, Tervuren, Belgium; Natural History Museum, London, United Kingdom; Royal Belgian Institute of Natural Sciences, Brussels, Belgium; Natural History Museum of Denmark, Copenhagen, Denmark; Naturalis Biodiversity Center, Leiden, the Netherlands. 